\title{
Transcriptome sequencing analysis of mono-ADP-ribosylation in colorectal cancer cells
}

\author{
NING-NING ZHANG ${ }^{*}$, TING LIN*, MING XIAO, QING-SHU LI, XIAN LI, \\ LIAN YANG, CHUAN-LING WANG and YA-LAN WANG \\ Department of Pathology, Molecular Medicine and Cancer Research Center, \\ Chongqing Medical University, Chongqing 400016, P.R. China
}

Received August 15, 2019; Accepted January 16, 2020

DOI: $10.3892 /$ or.2020.7516

\begin{abstract}
Colorectal cancer (CRC) is a global health concern. The role of epigenetics in tumors has garnered increasing interest. ADP ribosylation is an epigenetic modification that is associated with a variety of biological functions and diseases, and its association with tumor development and progression has been hypothesized. However, due to the limitations of available techniques and methods, ADP ribosylation of specific sites is difficult to determine. In previous studies, it was shown that arginine-117 of histone 3 (H3R117) in Lovo cells can be modified by mono-ADP-ribosylation. This site was mutated and Lovo cells overexpressing this mutant construct were established. In the present study, the expression
\end{abstract}

Correspondence to: Professor Ya-Lan Wang, Department of Pathology, Molecular Medicine and Cancer Research Center, Chongqing Medical University, 1 Yixueyuan Road, Yuzhong, Chongqing 400016, P.R. China

E-mail: wangyalan@cqmu.edu.cn

*Contributed equally

Abbreviations: CRC, colorectal cancer; KOG, eukaryotic orthologous group; DEGs, differentially expressed genes; RNA-seq, RNA sequencing; AE, alternative exon ends; SKIP, exon skipping; TSS, alternative transcription start site; MIR, multiple introns; TTS, alternative transcription termination site; GO, Gene Ontology; SNP, single nucleotide polymorphism; KEGG, Kyoto Encyclopedia of Genes and Genomes; MSKIP, cassette exons; IR, retention of single; TET, ten-eleven translocation; STRING, Search Tool for the Retrieval of Interacting Genes/Proteins; CENPBD1, CENPB DNA-binding domain containing 1; HIST2H3D, histone cluster 2 H3 family member D; CHST14, carbohydrate sulfotransferase 14; UST, uronyl 2-sulfotransferase; CHST15, N-acetyl galactosamine 4-sulfate 6-O-sulfotransferase; HIST2H3PS2, histone cluster 2 H3 pseudogene 2; HIST1H2AI, histone cluster $1 \mathrm{H} 2 \mathrm{~A}$ family member I; B3GALT6, galactosylxylosyl protein 3- $\beta$-galactosyltransferase; CHPF, chondroitin polymerizing factor; $\mathrm{CHPF} 2$, chondroitin polymerizing factor 2

Key words: CRC, epigenetic, histone modification, mono ADP ribosylation, transcriptome sequencing, differential gene expression of differentially expressed genes (DEGs) between untransfected Lovo cells and H3R117A Lovo cells was analyzed. A total of 58,174 DEGs were identified, of which 2,324 were significantly differentially expressed (q-value $<0.05$; fold change $>2$ ). Functional annotation and Kyoto Encyclopedia of Genes and Genomes pathway enrichment was used to analyze the functions and possible roles of the DEGs. The DEGs were enriched in pathways associated with metabolic process, catalytic activity, organelle and chromatin structure, and dynamics. Through this comprehensive and systematic analysis, the role of mono-ADP-ribosylation in CRC was examined, providing a foundation for future studies.

\section{Introduction}

Colorectal cancer (CRC) is the third most common type of cancer worldwide and the fourth most common cause of cancer-associated death $(1,2)$. Of newly diagnosed CRC cases, $5-25 \%$ of patients present with advanced-stage disease, and the prognosis for these patients remains poor (3).

ADP-ribosylation is an important post-translational modification. It is a reversible process mediated by ADP-ribosyltransferase and ADP-ribosylhydrolase. There are two forms of mono-ADP-ribosylation and poly-ADP-ribosylation (4,5). ADP-ribosylation involves the regulation of multiple biological processes, including different forms of stress response and metabolism (5). Increasing attention is being paid to the role of ADP ribosylation in tumors. Poly-ADP ribose polymerase inhibitors have recently entered clinical trials as anticancer agents $(5,6)$, and the FDA-approved inhibitors, olaparib, niraparib and rucaparib, are used for treatment of breast and ovarian cancer $(7,8)$. Studies have shown that MACRO domain containing 2 mono-ADP-ribosylhydrolase focal deletions are observed in human CRC $(9,10)$. The level of ADP-ribosylation of targets is dependent on the kinetics of turnover at the modification site (4). Numerous studies have focused on the identification of ADP ribosylation sites $(8,11,12)$, including arginine-specific mono-ADP-ribosyltransferase 1 (ARTC1) which can mono-ADP-ribosylate several arginine residues in the immune modulatory cationic peptides LL-37 and human neutrophil peptide-1 (4). ARTC1 in airway epithelial cells catalyzes arginine-14 of defensin-1 to reduce its antibacterial and cytotoxic activity (13). This suggests 
that ADP-ribosylation at different sites may exert different biological effects. Compared with poly-ADP-ribosylation, mono-ADP-ribosylation has not been studied as extensively. The mono-ADP-ribosylation modification sites of histones in human $\mathrm{CRC}$ cell lines with differing degrees of differentiation were screened in a previous study. Arginine-117 of histone H3 (H3R117) in human colon cancer Lovo cells, which are not substantially differentiated, was mono-ADP-ribosylated, and this promoted the proliferation of colon cancer cells (14).

Advances in high-throughput RNA sequencing (RNA-seq) techniques and bioinformatics methods have facilitated the sequencing of the entire human genome over the past decade (15), and are increasingly being used to identify novel targets for clinical use. Identifying drug-sensitive target genes using transcriptome sequencing may be used to better personalize treatments for each specific patient $(3,16,17)$.

In a previous study, it was shown that there was no significant difference in proliferation and apoptosis between Lovo cells transfected with an empty vector and untransfected cells. Lovo cells transfected with a H3R117A mutant construct and untransfected cells were thus used to analyze the effects of mono-ADP-ribosylation on the gene expression profiles of colon cancer cells by sequencing the transcriptomes of each cell type. The functions of these differentially expressed genes (DEGs) were analyzed using bioinformatics analysis, and Kyoto Encyclopedia of Genes of Genomes (KEGG) analysis of the DEGs showed that pathways associated with glycosaminoglycan biosynthesis-chondroitin sulfate/dermatan sulfate were significantly enriched. Studies have shown that the content and composition of glycosaminoglycan disaccharide are altered in CRC (18-20). Changes in the expression levels of associated enzymes, such as glycosyltransferases and sulfotransferases, also play an important role during the process of chondroitin sulfate biosynthesis. Additionally, chondroitin sulfate chains promote epithelial-mesenchymal transition $(21,22)$, suggesting that glycosaminoglycan biosynthesis-chondroitin sulfate/dermatan sulfate are closely associated with CRC metastasis. In the present study, the association between mono-ADP ribosylation on members of this pathway and the progression of CRC was examined, with the aim of providing a basis for further studying the role of mono-ADP-ribosylation in tumor development and progression.

\section{Materials and methods}

Cell samples. In a previous study, it was demonstrated that H3R117 is a mono-ADP-ribosylated site in Lovo cells, and Lovo cells with a point mutation resulting in H3R117A were successfully constructed [mutation of arginine at residue 117 of H3 to a non-ADP-ribosylated alanine (14)].The untransfected Lovo cells (donated by Professor Wei-Xue Tang, Chongqing Medical University) and H3R117A Lovo cells were both lysed with RNAiso Plus (Takara Bio, Inc.) and sent to Sangon Biotech Co., Ltd. Both types of cells were cultured in DMEM (HyClone; GE Healthcare Life Sciences) with 10\% FBS (HyClone; GE Healthcare Life Sciences) with $5 \% \mathrm{CO}_{2}$ at $37^{\circ} \mathrm{C}$.

Data evaluation and quality control. The raw image format obtained by Illumina Hiseq ${ }^{\mathrm{TM}}$ (Illumina, Inc.) via Casava (http://support.illumina.com/sequencing/sequencing_software/ casava.ilmn; version 1.8.2) base calling analysis was converted to raw sequenced reads, and termed raw data. The raw data quality value and other information were statistically analyzed, and the raw data quality of samples was visually evaluated using FastQC (http://www.bioinformatics. bbsrc.ac.uk/projects/fastqc/; version 0.11.2). Trimmomatic (http://www.usadellab.org/cms/index.php?page=trimmomatic; version 0.36 ) was used to filter the raw data to obtain the clean data by removing n-bases and removing the adaptor sequence in reads: Forward, AGATCGGAAGAGCACACGTCTGAAC and reverse AGATCGGAAGAGCGTCGTGTAGGGA. Low quality bases were removed from reads $3^{\prime}-5$ ' (q-value $<20$ ); low quality bases were removed from reads 5'-3' (Q-value <20); the sliding window method (23) was used to remove the bases with a mass value $<20$ in the tails of reads (window size, $5 \mathrm{bp}$ ); the reads and their matched reads with a length of $<35$ nucleotides were removed; 10,000 sequences were randomly extracted from the clean data and compared with the NCBI NT database (http://ncbi.nlm.nih.gov/) using blastn. E-value $\leq 1 \times 10^{-10}$ and comparison of results with a similarity $>90 \%$ and coverage $>80 \%$ were used to calculate species distribution and perform pollution detection.

RNA-seq evaluation. HISAT2 (version 2.1.0) was used to align the effective sample data to the reference genome, and statistical comparisons were performed using RseQC (http://rseqc. sourceforge.net/; version 2.6.1). RSeQC was used to analyze the duplicate reads and the distribution of inserted fragments based on the alignment results. Qualimap (http://www. qualimap.org; version 2.2.1) was used to test the gene body coverage and analyze the structural distribution of the genome. BEDTools (http://code.google.com/p/bedtools; version 2.26.0) was used for statistical analysis of gene coverage and read distribution on chromosomes.

Genetic structure analysis. BCFtools (http://samtools.sourceforge.net; version 1.5) was used to perform single nucleotide polymorphism (SNP)/insertion-deletion (indel) calling, and the SNPs/indels in each sample were extracted. Subsequently, SnpEff (snpeff.sourceforge.net; version 2.36) was used to calculate the distribution of variation sites on the genome structure; a quality value $>20$ and coverage cutoffs $>8$ were used as the criteria for filtering. Alternative splicing analysis was used for fusion gene analysis using ASprofier (http://ccb.jhu.edu/software/ASprofile; version 2.36) and EricScript (http://ericscript. sourceforge.net; version 0.55 ).

Expression level analysis. In the RNA-seq analysis, the expression levels of genes were estimated by counting the reads located in the genomic regions or gene exon regions. In addition to the true expression levels of the gene, the read count was positively correlated with the length and sequencing depth of the gene. To make the estimated gene expression levels of different genes and different experiments comparable, the concept of transcripts per million (TPM) was introduced. The calculation formula of TPM was as follows:

$$
\begin{gathered}
T P M_{i}=\frac{X_{i}}{L_{i}} * \frac{1}{\sum_{j} \frac{X_{j}}{L_{j}}} * 10^{6} \\
X_{i}=\text { total exon fragment } / \text { reads } \quad L_{i}=\frac{\text { exon length }}{K B}
\end{gathered}
$$


StringTie (http://ccb.jhu.edu/software/stringtie; version 1.3.3b) and known gene models (24) were used to assess gene expression.

Replicated correlation test. Biological replicates are necessary for any biological experiment. The correlation of gene expression levels between samples was used as an important index to test the reliability of the experiments and the rationality of sample selection. The closer the correlation coefficient was to 1 , the higher the similarity of expression patterns between samples. A total of three correlation indexes were calculated: Pearson, Kendall and Spearman (R software; version 3.1.2).

Expression difference analysis. DESeq2 (R package; https://github.com/mikelove/DESeq2; version 1.12.4) was used for gene expression difference analysis, and the results of the expression difference analysis were visualized. The DEGs were mapped to the Search Tool for the Retrieval of Interacting Genes/Proteins (STRING) protein interaction network database (http://string-db.org/), which was used to construct the protein interaction network. Based on the results of the differential analysis, Venn diagrams (VennDiagram R package; https://CRAN.R-project.org/package=VennDiagram; version 1.6.17) were drawn.

Gene enrichment analysis. TopGO (R package https://bioconductor.org/packages/topGO/; version 2.24.0) was used for Gene Ontology (GO) enrichment analysis, and the significance of GO-directed acyclic graph was drawn. ClusterProfiler (R package https://guangchuangyu.github.io/software/clusterProfiler; version 3.0.5) was used for KEGG pathway and eukaryotic orthologous group (KOG) classification enrichment analysis. The association analysis network diagram was drawn based on the results of the gene function enrichment analysis.

Western blotting. The cells were lysed in cell lysis buffer for western blotting (Beyotime Institute of Biotechnology) supplemented with PMSF (100:1; Beyotime Institute of Biotechnology) to extract the total proteins. The concentrations of the proteins were detected using a bicinchoninic acid protein assay kit (Beyotime Institute of Biotechnology). Proteins (20 $\mu \mathrm{g}$ per lane) were loaded on an SDS gel (10\%), resolved using SDS-PAGE and subsequently transferred to PVDF membranes (GE Healthcare Life Sciences), Membranes were blocked in 5\% non-fat milk for $2 \mathrm{~h}$ at room temperature and incubated with primary antibodies against $\beta$-actin (ProteinTech Group, Inc; cat. no. 66009-1-Ig; 1:20,000) or $\beta$-catenin (Wanleibio Co., Ltd.; cat. no. WL0962a; $1: 500)$ at $4^{\circ} \mathrm{C}$ overnight, and subsequently incubated with a HRP-conjugated secondary antibody [ProteinTech Group, Inc.; cat. nos. SA00001-1 (mouse) or SA00001-2 (rabbit); 1:5,000] for $2 \mathrm{~h}$ at room temperature. Signals were visualized using enhanced chemiluminescence reagent (EMD Millipore), and the protein level analysis was performed using ImageLab software (Bio-Rad Laboratories, Inc.; version 6.0.0).

RNA extraction and reverse transcription-quantitative PCR. Total RNA was extracted from cells using RNAiso Plus reagent (Takara Bio, Inc.). RNA was reverse transcribed to cDNA using an Evo M-MLV RT kit with gDNA Clean for qPCR (Hunan Accurate Bio-Medical Co., Ltd.), according to the manufacturer's protocol $\left(37^{\circ} \mathrm{C}\right.$ for $15 \mathrm{~min}, 85^{\circ} \mathrm{C}$ for $5 \mathrm{sec}$, then $\left.4^{\circ} \mathrm{C}\right)$. The primers were synthesized by Sangon Biotech Co., Ltd. The sequences of the primers were as follows (5'-3'): Galactosylxylosyl protein 3- $\beta$-galactosyltransferase (B3GALT6) forward, CGACGCCTACGAAAACCTCA and reverse, GTCGTACACGTAGGACAGGC; N-acetyl galactosamine 4-sulfate 6-O-sulfotransferase (CHST15) forward, GCC ACTCAATGCCATCCAGA and reverse, ATGGCAGGCTCG AGAACCAC; chondroitin 4-sulfotransferase 2 (CHST12) forward, CGACGAGTTTCTGGACAAG and reverse, GGA CCAGTCGTAGCCTCT; and $\beta$-actin forward, AGCGAGCAT CCCCCAAAGTT and reverse GGGCACGAAGGCTCATCA TT. qPCR was performed using SYBR ${ }^{\circledast}$ Premix Ex Taq ${ }^{\mathrm{TM}}$ II, according to the manufacturer's protocol (Takara Bio, Inc.) as follows: $95^{\circ} \mathrm{C}$ for $30 \mathrm{sec}, 95^{\circ} \mathrm{C}$ for $5 \mathrm{sec}, 60^{\circ} \mathrm{C}$ for $30 \mathrm{sec}$ for a total of 40 cycles, followed by $95^{\circ} \mathrm{C}$ for $10 \mathrm{sec}$, and melting curve analysis between 65 and $95^{\circ} \mathrm{C}$ (increments of $0.5^{\circ} \mathrm{C}$ ) for $5 \mathrm{sec}$. $\beta$-actin was used as the reference to normalize the results and the data were analyzed using the $2^{-\Delta \Delta \mathrm{Cq}}$ method (25).

Immunofluorescence. Cells $\left(1 \times 10^{5} / \mathrm{ml}\right)$ were fixed in $4 \%$ paraformaldehyde for $20 \mathrm{~min}$ at room temperature and permeabilized using $0.1 \%$ Triton X-100 for $20 \mathrm{~min}$. Samples were blocked in goat serum (Wuhan Boster Biological Technology, Ltd.; cat. no. AR009) for 30 min and subsequently incubated with the following primary antibodies: Anti- $\beta$-catenin (Wanleibio Co., Ltd.; cat. no. WL0962a; 1:200) and anti-mono-ADP-ribose binding reagent (EMD Millipore; cat. no. MABE1076; 1:200) at $4^{\circ} \mathrm{C}$ overnight. The following day, cells were washed in PBS and incubated with Cy3-conjugated secondary antibody (ProteinTech Group, Inc.; cat. no. SA00009-2; 1:200) in the dark for $1 \mathrm{~h}$ at room temperature. DAPI (Wuhan Boster Biological Technology, Ltd.) was used for nuclear staining (5 min at room temperature). The images were captured using ZOETM Fluorescent Cell Imager (Bio-Rad Laboratories, Inc.) and analyzed using ImageJ software (version 1.48; National Institutes of Health) The experiment was repeated three times.

Wound healing assays. Cells were seeded in 6-well plates. When the cell density reached $90 \%$, the monolayers were scratched using a $200 \mu 1$ sterile pipette tip, and the cells were cultured in serum-free medium for $24 \mathrm{~h}$ at $37^{\circ} \mathrm{C}$. The wounded monolayers were imaged using a ZOETM Fluorescent Cell Imager (Bio-Rad Laboratories, Inc.) at the start of the experiment and after $24 \mathrm{~h}$. The experiment was repeated three times and the images were analyzed using Image J version 1.48 (National Institutes of Health).

Transwell assay. Transwell assays were performed using Transwell chambers (Merck KGaA) coated with Matrigel (Corning, Inc.). Serum-free cell suspension with $5 \times 10^{5}$ cells were added to the upper chamber of the well at $200 \mu \mathrm{l} / \mathrm{chamber}$, whereas supplemented medium with $10 \%$ serum was added to the lower chamber. After $24 \mathrm{~h}$ of incubation at $37^{\circ} \mathrm{C}$, the cells were fixed with $4 \%$ paraformaldehyde for $30 \mathrm{~min}$ and stained with $0.1 \%$ crystal violet for $20 \mathrm{~min}$ at room temperature, followed by washing three times. Images were captured (magnification, x200) under a light microscope (Nikon Corporation), the experiment was repeated three times, and the number of cells was counted using ImageJ version 1.48 (National Institutes of Health). 
Table I. Raw data statistics.

\begin{tabular}{lcc}
\hline Statistic & Non_transfection & H3_R117A \\
\hline Total Reads Count (n) & 48337200 & 47928086 \\
Total Bases Count (bp) & 7250580000 & 7189212900 \\
Average Read Length (bp) & 150 & 150 \\
Q10 Bases Count (bp) & 7250443310 & 7189118632 \\
Q10 Bases Ratio (\%) & $100.00 \%$ & $100.00 \%$ \\
Q20 Bases Count (bp) & 7029222588 & 6953852019 \\
Q20 Bases Ratio (\%) & $96.95 \%$ & $96.73 \%$ \\
Q30 Bases Count (bp) & 6666265867 & 6566925897 \\
Q30 Bases Ratio (\%) & $91.94 \%$ & $91.34 \%$ \\
N Bases Count (bp) & 136690 & 94268 \\
N Bases Ratio (\%) & $0.00 \%$ & $0.00 \%$ \\
GC Bases Count (bp) & 3700903258 & 3616005152 \\
GC Bases Ratio (\%) & $51.04 \%$ & $50.30 \%$ \\
\hline
\end{tabular}

$\mathrm{H} 3$, histone $\mathrm{H} 3$.

Table II. Quality control data statistics.

\begin{tabular}{lcc}
\hline Statistic & Non_transfection & H3_R117A \\
\hline Total Reads Count (n) & 46445980 & 45973604 \\
Total Bases Count (bp) & 6786715331 & 6720275957 \\
Average Read Length (bp) & 146.12 & 146.18 \\
Q10 Bases Count (bp) & 6786671778 & 6720232139 \\
Q10 Bases Ratio (\%) & $100.00 \%$ & $100.00 \%$ \\
Q20 Bases Count (bp) & 6670691000 & 6589709477 \\
Q20 Bases Ratio (\%) & $98.29 \%$ & $98.06 \%$ \\
Q30 Bases Count (bp) & 6375757096 & 6272795618 \\
Q30 Bases Ratio (\%) & $93.94 \%$ & $93.34 \%$ \\
N Bases Count (bp) & 43553 & 43818 \\
N Bases Ratio (\%) & $0.00 \%$ & $0.00 \%$ \\
GC Bases Count (bp) & 3466686895 & 3381728755 \\
GC Bases Ratio (\%) & $51.08 \%$ & $50.32 \%$ \\
\hline
\end{tabular}

H3, histone $\mathrm{H} 3$.

Statistical analysis. DEGs were determined using a negative binomial distribution, and enrichment analysis was performed using a hypergeometric distribution. DESeq2 was used to analyze differences in gene expression difference. SnpEff was used to calculate the distribution of variation sites in the genome structure, and the filters used were mass value $>20$ and coverage $>8$. Variable shear events were classified using ASprofile based on the predicted gene model for each sample. TopGO was used for GO enrichment analysis and ClusterProfiler was used for KEGG pathway and KOG enrichment analysis. All experiments were performed three times and the data are presented as the mean \pm standard deviation. Statistical analysis was performed using GraphPad Prism version 5 (GraphPad Software, Inc.). An unpaired two-tailed Students' t-test was used to compare groups. $\mathrm{P}<0.05$ was considered to indicate a statistically significant difference.

\section{Results}

Confirmation of mono-ADP-ribosylation in Lovo cells. A previous study identified mono-ADP-ribosylation of H3R117 in Lovo cells using liquid chromatography with tandem mass spectrometry (LC-MS/MS) (14). In the present study, immunofluorescence experiments were performed to confirm the mono-ADP-ribosylation in these cells. Mono-ADP-ribosylation was notably present in Lovo cells, particularly in the nucleus (Fig. S1). These results demonstrated that nuclear mono-ADP-ribosylation is present in CRC.

Preprocessing of raw data. FastQC was used to visually evaluate the quality of the raw data (Fig. 1) and Trimmomatic was used to filter the raw data to obtain the clean data. Data information prior to and following quality control showed Q20 $>90$ and Q30 >90\% (Tables I and II). These results showed that the experimental data was of high quality, and the stability of the instrumental analysis systems was good and met the requirements for subsequent analysis.

RNA-seq evaluation. Certain important quality metrics can only be measured when reads are matched with the reference genome and their positions are aligned to the annotation information, including sequencing saturation, distribution of reads with different genome characteristics and coverage uniformity of transcripts.

The reference genome was used as the reference sequence, HISAT2 was used to map the quality-controlled sequencing reads to the reference genome, and statistical comparisons were performed using RSeQC; 97\% of clean reads were mapped to the reference genome (Table III). Duplicate reads were defined as sequences with exactly the same base arrangement. Duplicate reads are primarily the result of PCR amplification during library construction. As shown in Fig. 1A and B, the overall trend of the curve showed a linear smooth extension and diffusion. As the horizontal axis increased, the corresponding vertical axis decreased, suggesting that the results were normal. Ideally, for RNA-seq, there should be independent sampling between sequencing reads, and the distribution of reads on all expressed transcripts should be homogenized. The analysis of gene coverage from 5'-3' is an indicator used to evaluate the randomness of transcriptome sequencing (26). By mapping the results, the coverage of each transcriptional site was calculated, and the coverage rate of each transcriptional site was calculated. By calculating the coverage ratio of each gene in each sample, the proportion of all the samples sequenced completely detected and the proportion of genes not detected was calculated, and could be used to determine whether there were genes with specific expression patterns among the samples (Fig. 1C).

Structural variation. RNA-seq can be used detect various structural variations in mRNA. In the present study, SNPs and alternative splicing (AS) were primarily analyzed. SNP analysis showed that the untransfected cells possessed 60,362 SNP sites and 3,979 indel sites. The cells expressing the mutant H3 possessed 54,918 SNP sites and 3,876 indel sites (Fig. 2A). Of the SNPs, $>50 \%$ were present in introns (Fig. 2D and E). SNPs included transition and transversion, and the majority 
Table III. Mapping results statistics.

\begin{tabular}{lcc}
\hline Statistic & H3_R117A, n (\%) & Non_transfection, $(\%)$ \\
\hline Total reads & $45836984(100.00)$ & $46315408(100.00)$ \\
Total mapped & $44785927(97.71)$ & $45140891(97.46)$ \\
Multiple mapped & $1150668(2.51)$ & $1213377(2.62)$ \\
Uniquely mapped & $43635259(95.20)$ & $43927514(94.84)$ \\
Read-1 mapped & $21913703(47.81)$ & $22096074(47.71)$ \\
Read-2 mapped & $21721556(47.39)$ & $21831440(47.14)$ \\
Reads mapping to ‘+' & $21829356(47.62)$ & $21966410(47.43)$ \\
Reads mapping to ‘-' & $21805903(47.57)$ & $21961104(47.42)$ \\
Non-splice reads & $24550439(53.56)$ & $24395895(52.67)$ \\
Splice reads & $19084820(41.64)$ & $19531619(42.17)$ \\
Reads mapped in proper pairs & $42549038(92.83)$ & $42585412(91.95)$
\end{tabular}
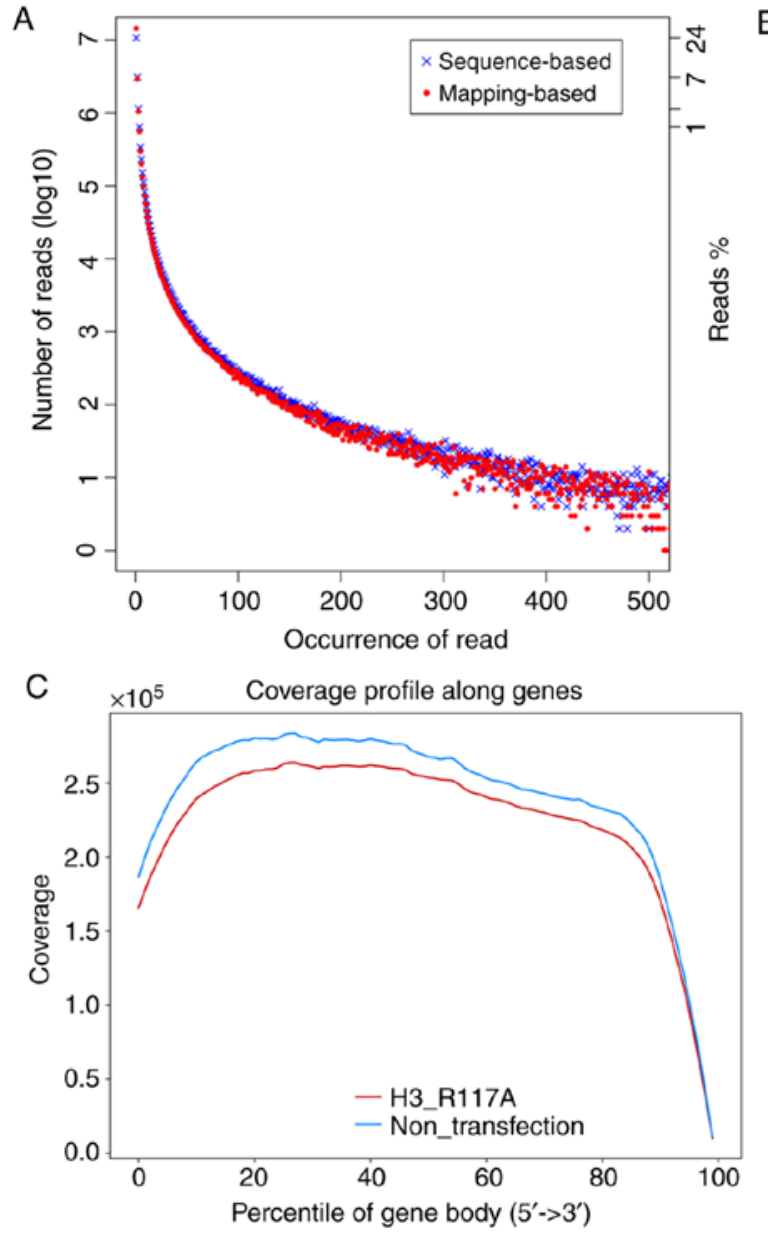

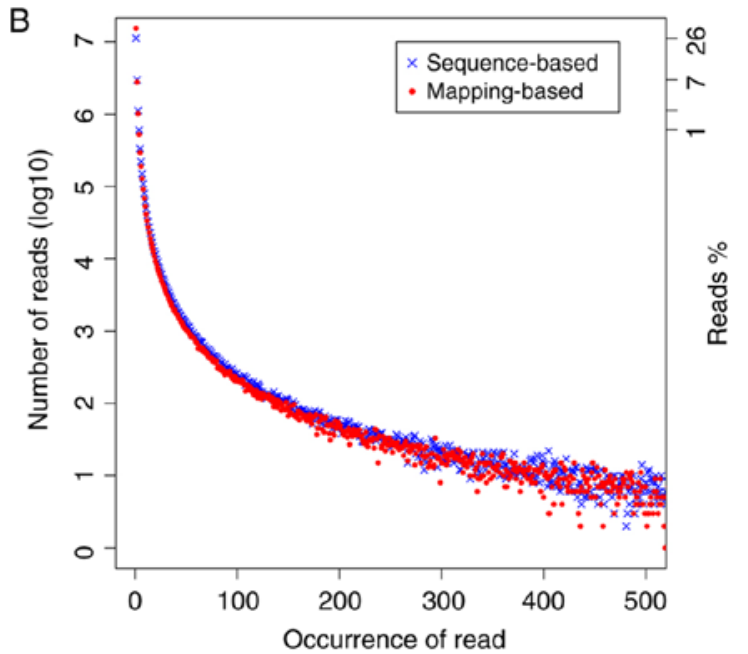

Figure 1. Diagram showing the distribution frequency of duplicate reads in (A) untransfected Lovo cells and (B) Lovo cells with the mutant H3R117A; the horizontal axis represents the frequency of duplicate reads, and the vertical axis represents the number of duplicate reads that were different at a certain frequency $(\log 10)$. Crosses represent the duplicate analysis based on all sequencing reads; dots represent the duplicate analysis based on the mapping of the sequencing reads to the reference sequence. The overall trend of the curve was used to evaluate the content and proportion of the duplicate reads obtained by sequencing. (C) Gene body coverage curves; the horizontal axis represents the base length of a single gene as a percentage, 0 represents the 5 ' terminus and 100 represents the $3^{\prime}$ terminus. The vertical axis represents the sum of the number of reads mapping to the horizontal axis of all genes. Each color represents a sample. The curve reflects whether the sequenced reads were evenly distributed on the genes. H3, histone 3 .

of the SNPs in both groups of cells were transitions. A total of four transition types were detected: $\mathrm{A}>\mathrm{G}, \mathrm{C}>\mathrm{T}, \mathrm{G}>\mathrm{A}$ and $\mathrm{T}>\mathrm{C}$. Among these, $\mathrm{A}>\mathrm{G}$ and $\mathrm{T}>\mathrm{C}$ were the most common.
Transversion was relatively rarely observed, but eight types of transversion were detected: $\mathrm{A}>\mathrm{C}, \mathrm{A}>\mathrm{T}, \mathrm{C}>\mathrm{A}, \mathrm{C}>\mathrm{G}, \mathrm{G}>\mathrm{C}, \mathrm{G}>\mathrm{T}$, $\mathrm{T}>\mathrm{A}$ and $\mathrm{T}>\mathrm{G}$ (Fig. $2 \mathrm{~B}$ and $\mathrm{C}$ ). Between the two cell types 

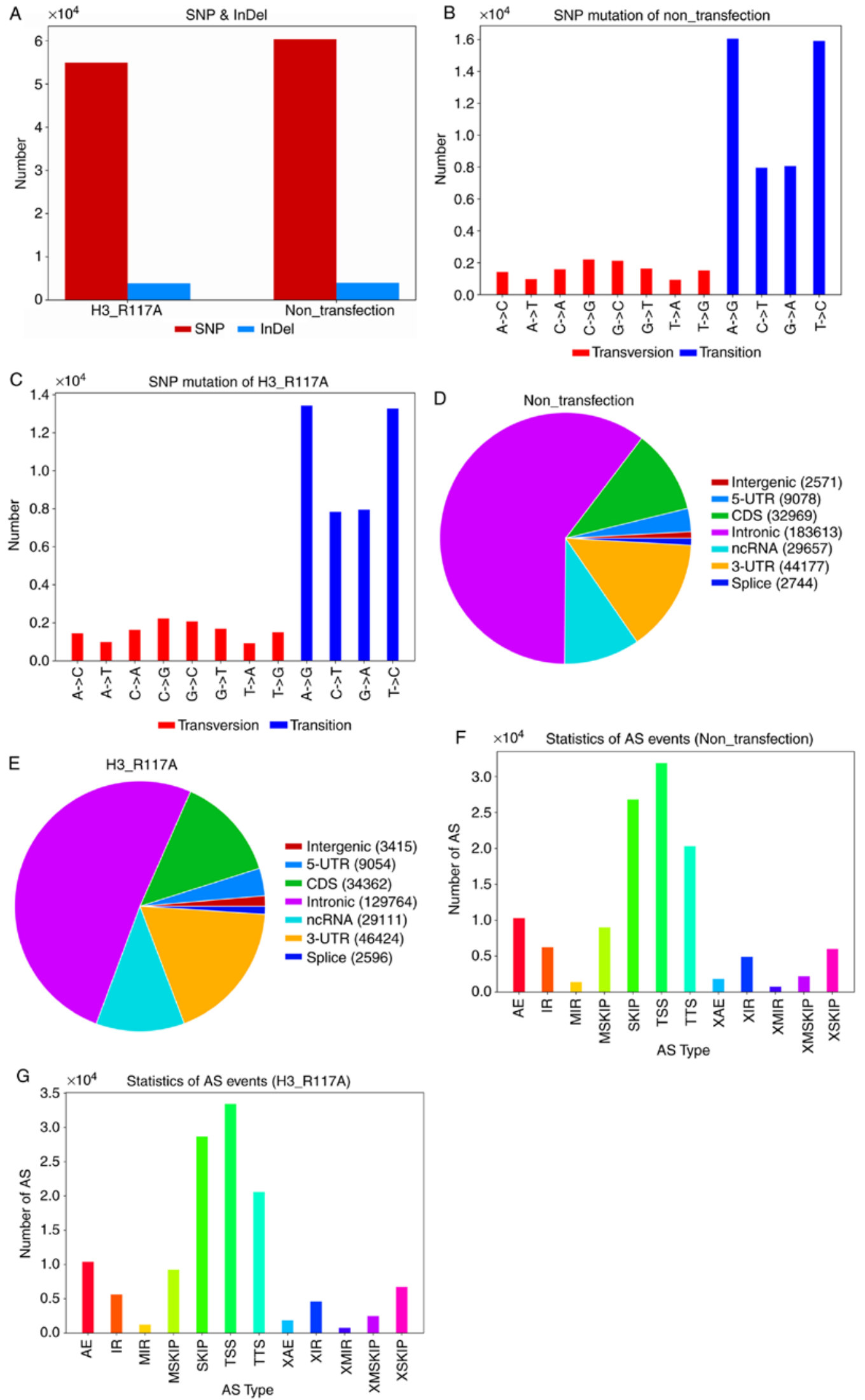

Figure 2. (A) Number of SNPs; the horizontal axis represents the sample name, and the vertical axis represents the number of variations. (B) Types of SNPs in untransfected Lovo cells; (C) types of SNPs in the mutant H3R117A; the horizontal axis represents the type of SNP, and the vertical axis the number of variations. (D) SNP effect region in untransfected Lovo cells; (E) SNP effect region in the mutant H3R117A; different colors represent different genetic elements, and the area occupied by each color corresponds to the percentage of variation of the element in the total variation. (F) Type of AS in untransfected Lovo cells; (G) Type of AS in in the mutant H3R117A; the horizontal axis represents the AS classification form, and the vertical axis represents the number of AS events. SNP, single nucleotide polymorphism; AS, alternative splicing; UTR, untranslated region; ncRNA, non-coding RNA; CDS, coding sequence; AE, alternative exon ends; IR, retention of single; MIR, multiple introns; MSKIP, cassette exons; SKIP, exon skipping; TSS, alternative transcription start site; TTS, alternative transcription termination site; XAE; XIR; XMIR; XMSKIP; XSKIP; H3, histone H3. 
assessed, the same gene exhibited different forms of AS, which greatly increases the capacity for gene encoding, as well as the complexity of gene expression and the diversity of protein function. Abnormal AS may produce potentially harmful or beneficial effects in the organism under certain environmental conditions. In the present study, ASprofile was used to classify AS according to the predicted gene models of each sample. The classification was as follows: SKIP, exon skipping; MSKIP, cassette exons; IR, retention of single; MIR, multiple introns; AE, alternative exon ends; TSS, alternative transcription start site; and TTS, alternative transcription termination site. The three types of AS most commonly observed in both cell groups were TSS, SKIP and TTS (Fig. 2F and G).

Biological replicates. A total of three correlation indices were calculated in the scatterplot of the replicated correlation test: Pearson $=0.8294$, Kendall $=0.8258$ and Spearman $=0.8911$. These correlation indices were all close to 1 , and the scatterplot showed that the majority of the points were centered around the diagonal line. These results indicated that the similarity of expression patterns between samples was high (Fig. 3).

Differential expression of genes. In total, 58,174 differentially expressed genes were identified; among these, 2,324 genes were significantly different (q-value $<0.05$; fold change $>2$ ). Compared with the untransfected cells, 1,391 upregulated genes and 933 downregulated genes were identified. DEGs were mapped using the STRING protein interaction network database to construct protein interaction networks (Fig. 4A). Based on the results of the differential analysis, Venn diagrams were drawn. The Venn diagram showed the number of expressed genes that were common and unique between the two groups (TPM >0). A total of 21,669 genes were present in both groups; 2,400 genes were unique to the $\mathrm{N}$ group, and 3,848 genes were unique to the mutant cells (Fig. 4B).

Functional enrichment analysis of DEGs. GO is an internationally standardized functional classification system for genes, providing a set of regularly updated descriptions to comprehensively describe the properties of genes and gene products in organisms. There are three ontologies in GO: Molecular function, cellular component and biological process (Fig. 5A). According to the results of the GO analysis, it was identified that for biological process, DEGs were significantly enriched in 'metabolic process' and 'negative regulation of biological process' (q-value $<0.05$ ); for cell component, DEGs were significantly enriched in 'organelle', 'organelle part' and 'membrane-enclosed lumen' (q-value <0.05); for molecular function, DEGs were significantly enriched in 'binding' and 'catalytic activity' (q-value <0.05; Fig. 5B).

Clusters of Orthologous Groups of proteins (COG) and KOG are the NCBI annotation system based on direct homologous relationships of genes. Among these, COG targets prokaryotes and KOG targets eukaryotes. COG/KOG combines evolutionary relationships, homologous genes from different species are divided into different Ortholog clusters. Currently, there are 4,873 categories for COG and 4,852 categories for KOG. The genes from the same ortholog have the same functions. In the present study, KOG classification indicated that the DEGs were classed into 26 functional categories. The only

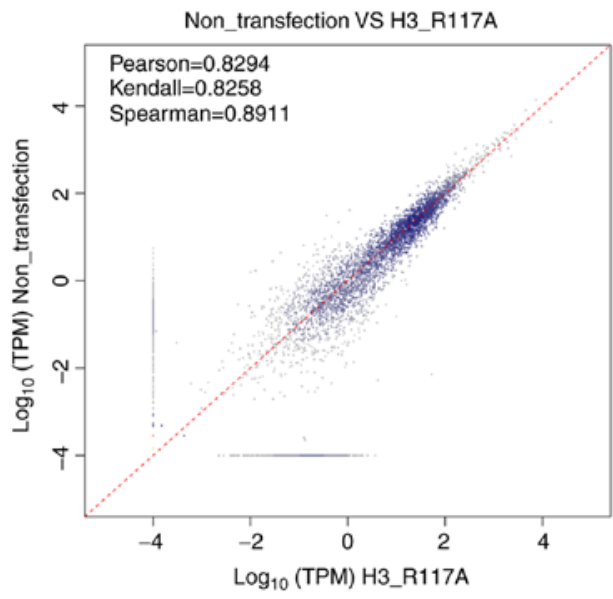

Figure 3. Gene TPM replicate correlation; the horizontal and vertical axes represent $\log 10$ TPM values of the two samples. Three correlation indices were calculated for this figure: Pearson, Kendall and Spearman. TPM, transcripts per million; $\mathrm{H} 3$, histone $\mathrm{H} 3$.

categories that were statistically significant were 'chromatin structure' and 'dynamics' (q-value <0.05). A total of 43 genes involved in the functional category were identified. Compared with the untransfected group, 14 genes were downregulated and 29 genes were upregulated. The top four genes with a $\log 2$ FoldChange were: Histone cluster $2 \mathrm{H} 3$ pseudogene 2 (HIST2H3PS2) (log2FoldChange=22.65), histone cluster 2 H3 family member D (HIST2H3D) ( $\log 2$ FoldChange=5.35), CENPB DNA-binding domain containing 1 (CENPBD1) ( $\log 2$ FoldChange $=2.84)$, histone cluster $1 \mathrm{H} 2 \mathrm{~A}$ family member I (HIST1H2AI) (log2FoldChange=2.78; Fig. 5C).

KEGG enrichment analysis. KEGG is a relatively complete database of biological systems, integrating genomic, chemical and system functional information. The pathway database is the most commonly used sub-database (27). In RNA-seq, KEGG enrichment was used to analyze the DEGs, which were shown as scatter plots (Fig. 6A). KEGG enrichment was measured using the Rich factor, q-value and the number of genes enriched in this pathway. A total of $306 \mathrm{KEGG}$ pathways were identified, and the top three most significant pathways are presented in Fig. 6B-D. These were 'glycosaminoglycan biosynthesis-chondroitin sulfate/dermatan sulfate' (ko00532), 'ubiquitin mediated proteolysis' (ko04120) and 'bladder cancer' (ko05219). Furthermore, the levels of expression of carbohydrate sulfotransferase 14 (CHST14), chondroitin 4-sulfotransferase 2 (CHST12), uronyl 2-sulfotransferase (UST), B3GALT6, chondroitin polymerizing factor 2 (CHPF2) were upregulated, whereas CHST15 and chondroitin polymerizing factor (CHPF) were downregulated in the glycosaminoglycan biosynthesis-chondroitin sulfate/dermatan sulfate pathway. RT-qPCR was used to confirm the changes in the top three genes based on the $\log 2$ FoldChange values. These were CHST12 ( $\log 2$ FoldChange=3.61), B3GALT6 (log2FoldChange=2.34) and CHST15 (log2FoldChange=-2.41) $(\mathrm{P}=0.0075)($ Fig. S2). The RT-qPCR results were consistent with the sequencing results. Similarly, the levels of expression of certain genes changed significantly in the ubiquitin-mediated proteolysis pathway and bladder cancer pathway. 
A
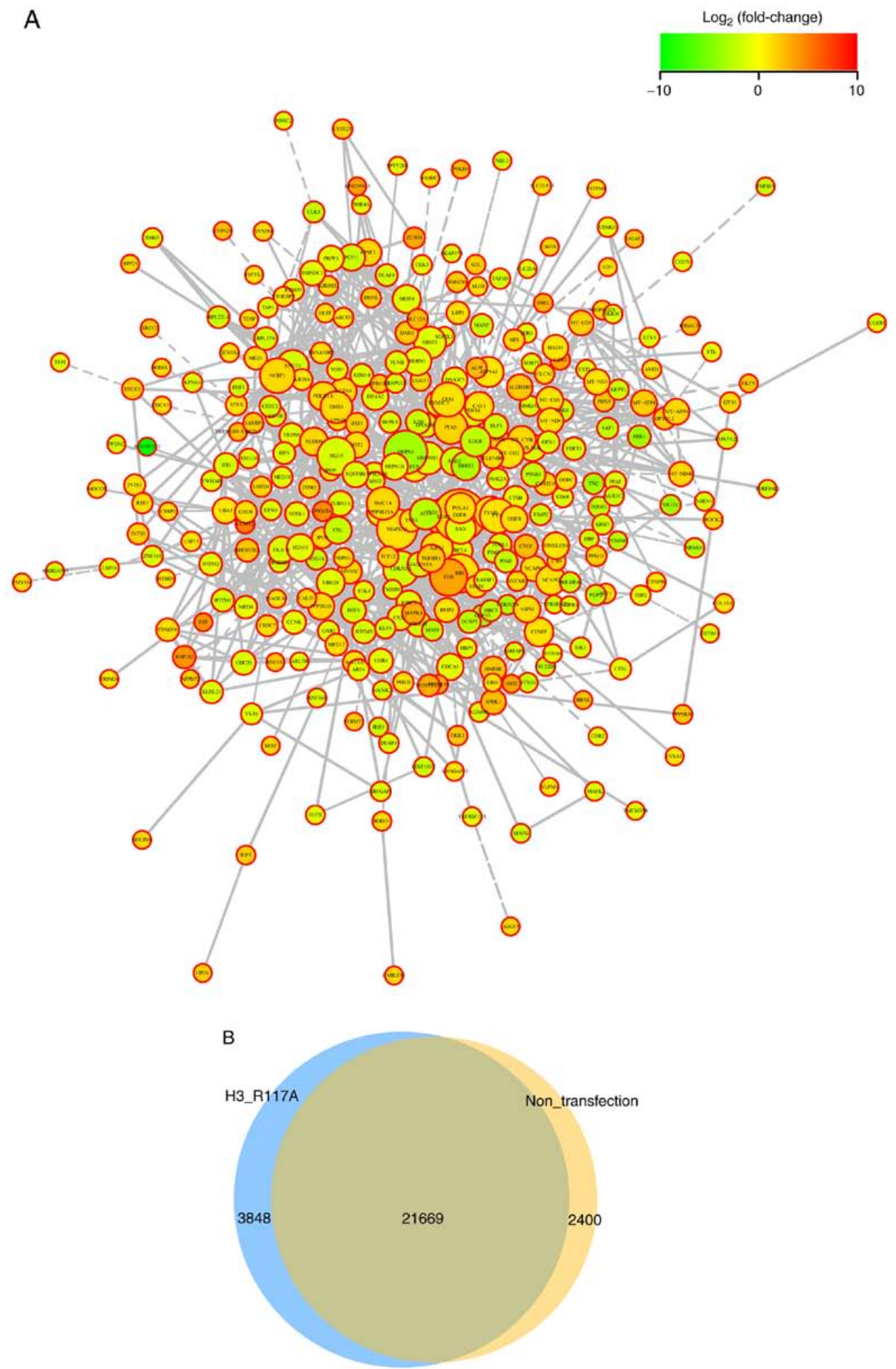

Figure 4. (A) Differential gene protein-protein interaction networks; nodes represent genes and edges represent interactions between two genes. The size of the node is proportional to the degree of connectivity of the node; that is, the more edges connected to the node, the larger the node is, indicating that the importance of the gene in the network. The node color represents the degree of expression difference of genes in the samples. Green represents downregulation and red represents upregulation, and the color depth represents the degree of differential regulation. The solid lines indicate that the relationships between genes have been verified, and the dotted lines indicate that they have not been verified. (B) Gene TPM Venn diagram; different samples are shown in different colors, and the numbers in the figure represent the number of unique or commonly expressed genes. The overlapping region represents the number of common genes, whereas the non-overlapping region represents the number of expressed genes unique to different samples. TPM, transcripts per million; H3, histone $\mathrm{H} 3$.

Mono-ADP-ribosylation of H3RI17 affects invasion and metastasis by mediating Wnt/ $\beta$-catenin signaling in Lovo cells. To examine the function of mono-ADP-ribosylation in invasion and metastasis in CRC, Transwell invasion and wound healing assays were performed. The results demonstrated that compared with the untransfected cells, the cell invasion 


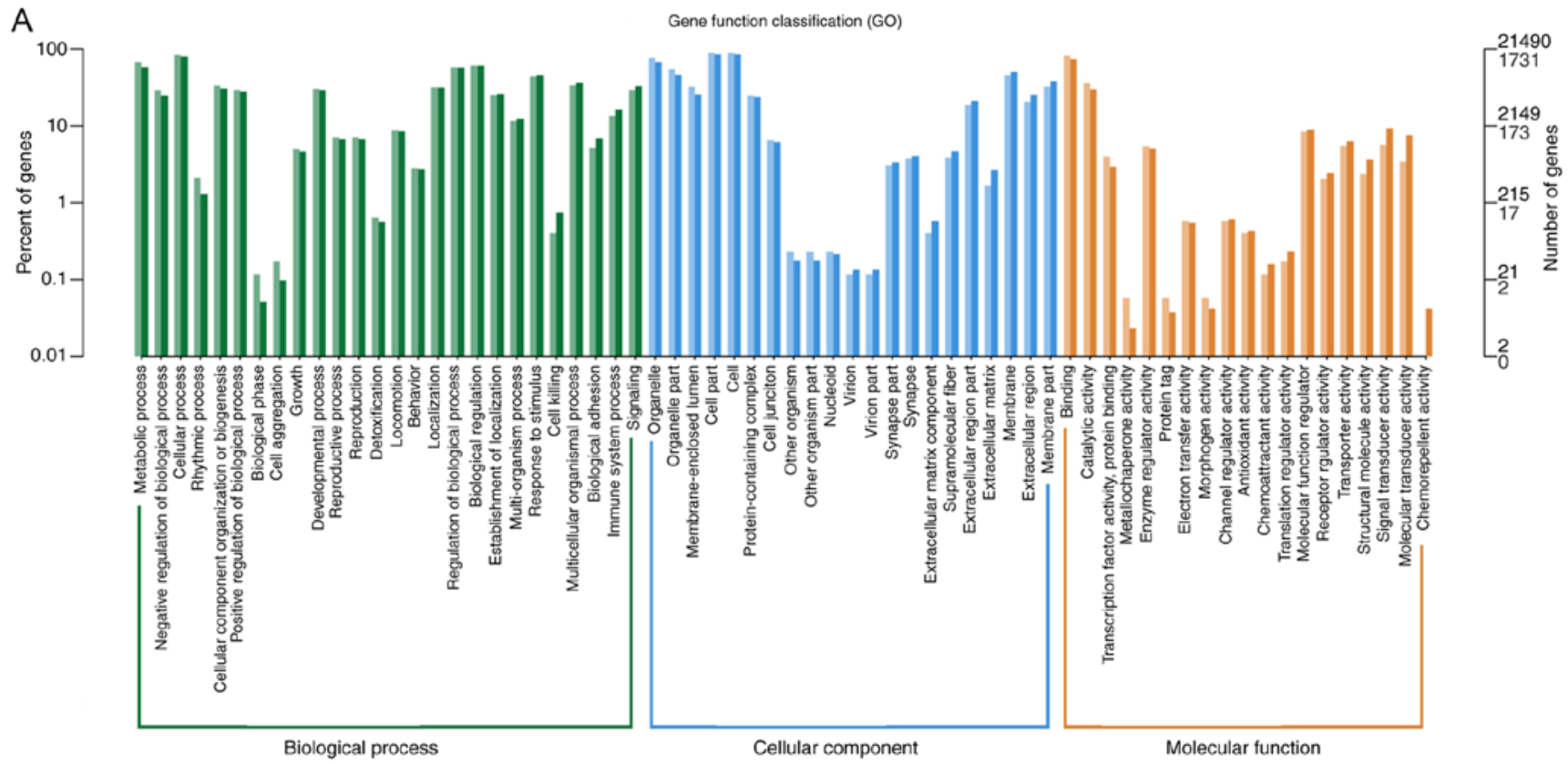

B GO Enrichment

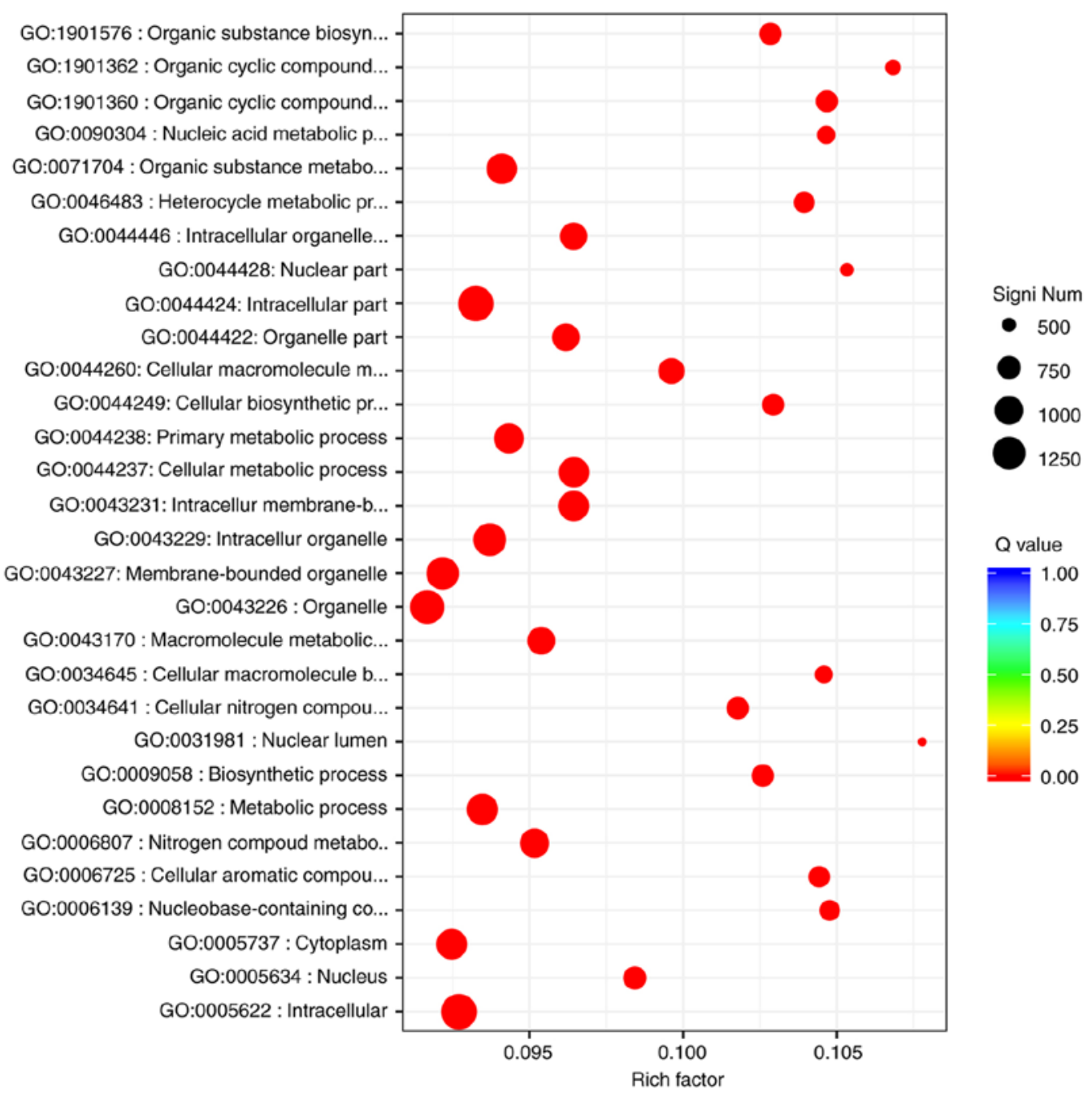

Figure 5. (A) GO categories; the horizontal axis is the functional classification, and the vertical axis is the number of genes in the classification (right) and the percentage of the total number of genes annotated (left). Different colors represent different categories. On the histograms and axes, light colors represent the differentially expressed genes, and dark colors represent all genes. (B) GO enrichment scatter; the vertical axis represents the functional annotation information, and the horizontal axis represents the Rich factor, corresponding to the function (the number of differentially expressed genes annotated to this function divided by the number of genes annotated to this function). The size of the q-value is represented by the colors of the dots. The smaller the q-value is, the closer the color is to red. Only the top $30 \mathrm{GO}$ terms with the highest degree of enrichment were analyzed. 
C
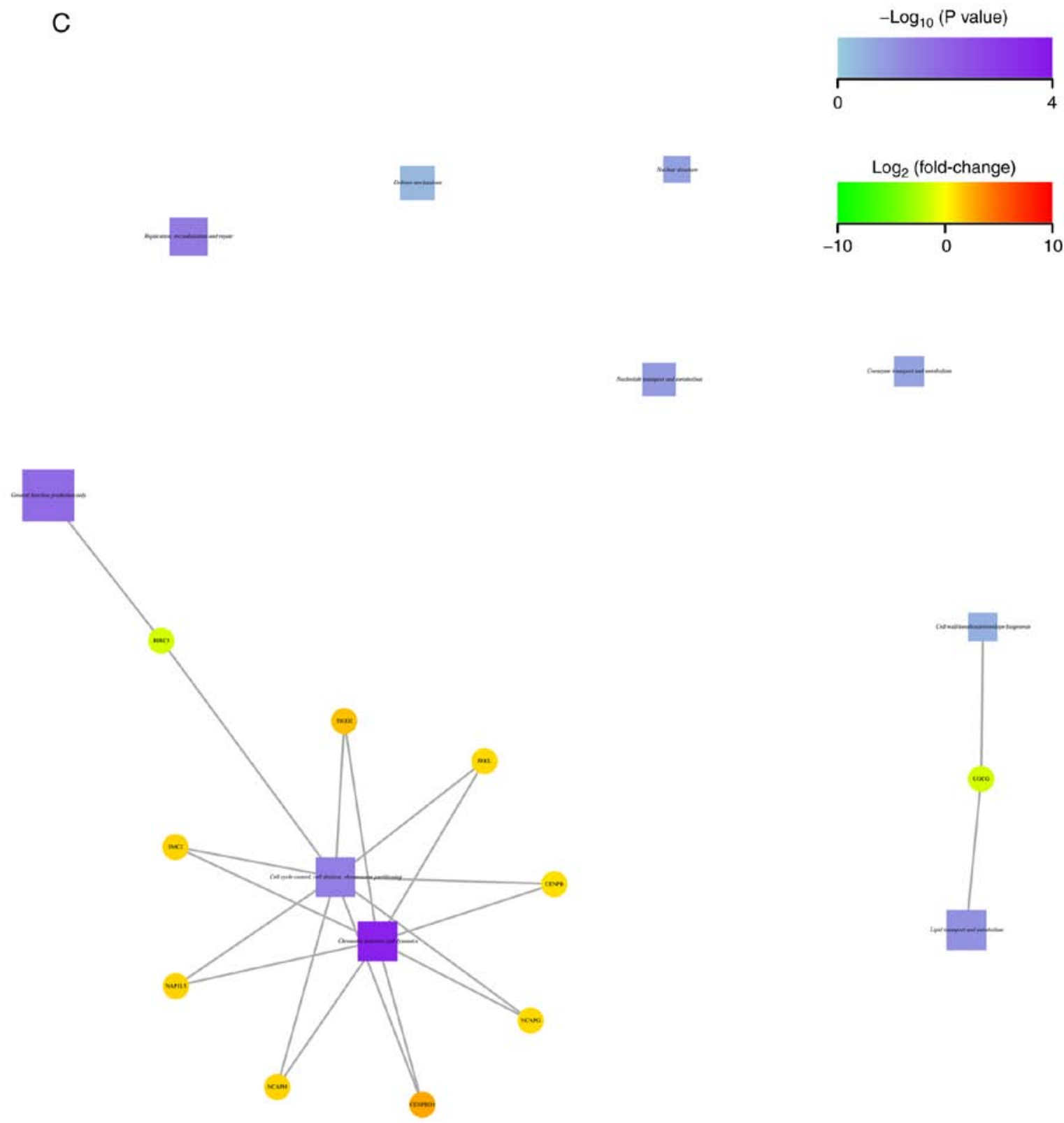

Figure 5. Continued. (C) KOG enrichment network; square nodes represent functional information, circular nodes represent genes, and edges represent the correlation between genes and functions. The size of the node is proportional to the degree of connectivity of the node; that is, the more edges there are connected with the node, the larger the node is. The color of circular nodes represents the expression difference of genes in the samples; green represents downregulation, red represents upregulation, and the color depth represents the differential regulation. The color of the square nodes represents the P-value. The higher the enrichment degree is, the lower the P-value is, and the darker the color is. The larger the square node area is, the more differentially expressed genes are involved and contribute to the biological phenomenon. Only the top 10 functions with the highest degree of enrichment and their differentially mapped genes were analyzed. GO, gene ontology; KOG, eukaryotic orthologous group.

and metastatic capacity $(\mathrm{P}=0.0105)$ of the mutated cells were reduced (Fig. S3A and B). Previous studies have shown that the glycosaminoglycan biosynthesis-chondroitin sulfate/dermatan sulfate pathway is associated with invasion and metastasis. Thus, how this pathway was involved in tumor metastasis was assessed. Previous studies have reported that chondroitin sulfate/dermatan sulfate proteoglycans interact with Wnt, resulting in increased accumulation of $\beta$-catenin, which regulates tumor metastasis and other biological activities (28). In the present study, western blotting and immunofluorescence were performed to evaluate the expression of $\beta$-catenin, and the results showed that the expression of $\beta$-catenin was decreased significantly in the mutation group $(\mathrm{P}=0.0138$ and $\mathrm{P}=0.0023$;
Fig. S3C and D, respectively). These results suggested that mono-ADP-ribosylation of H3R117 in Lovo cells regulates the biosynthesis of chondroitin sulfate/dermatan sulfate, and that this is involved in tumor metastasis by regulating $\mathrm{Wnt} / \beta$-catenin signaling.

\section{Discussion}

Chromatin modification can affect various biological processes, such as the compaction and plasticity of chromatin, gene expression, cell differentiation and apoptosis $(29,30)$. Histone $\mathrm{H} 3$ is the histone with the highest degree of post-translational modifications, and this includes ADP ribosylation (31). 
A

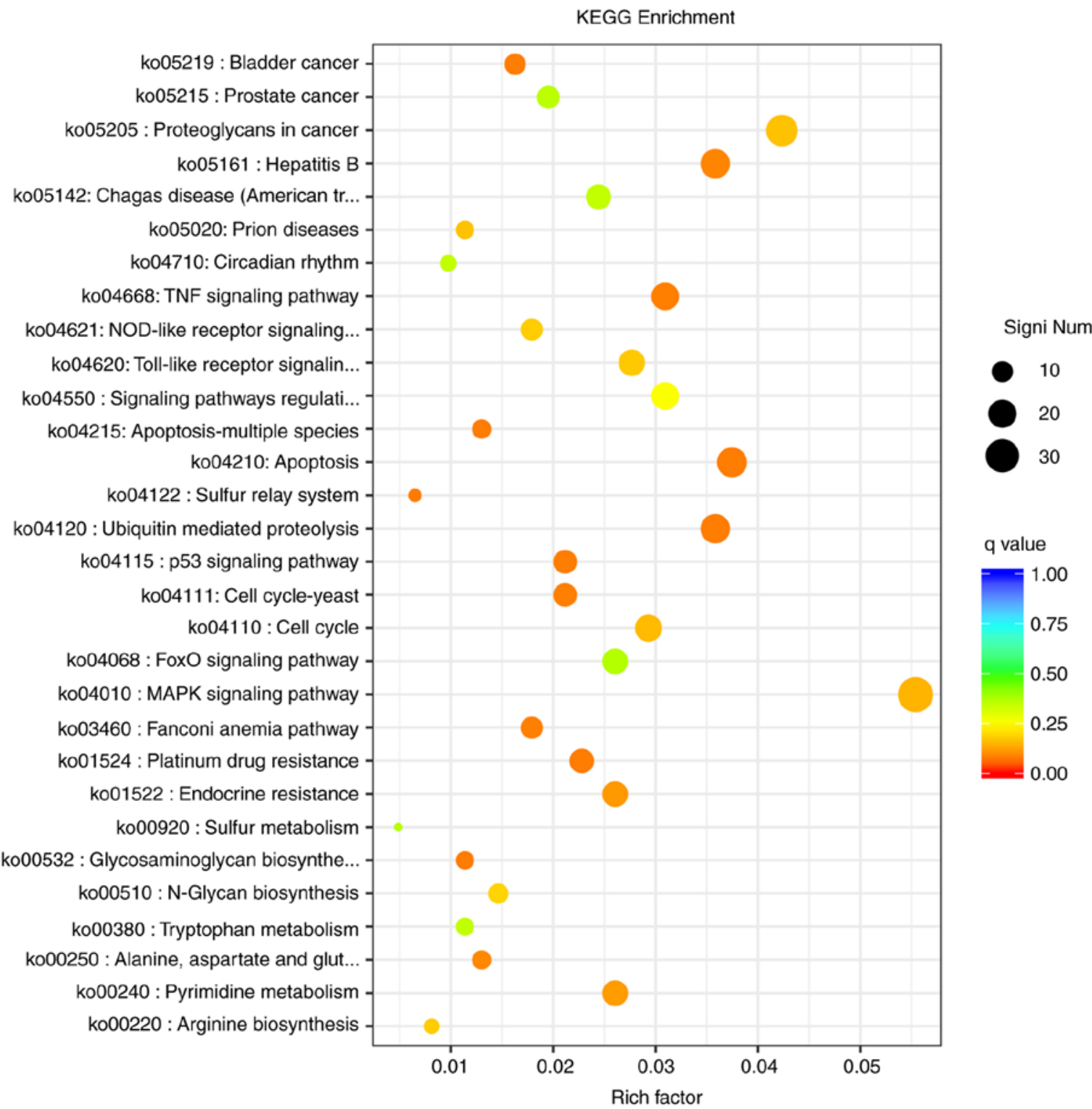

B

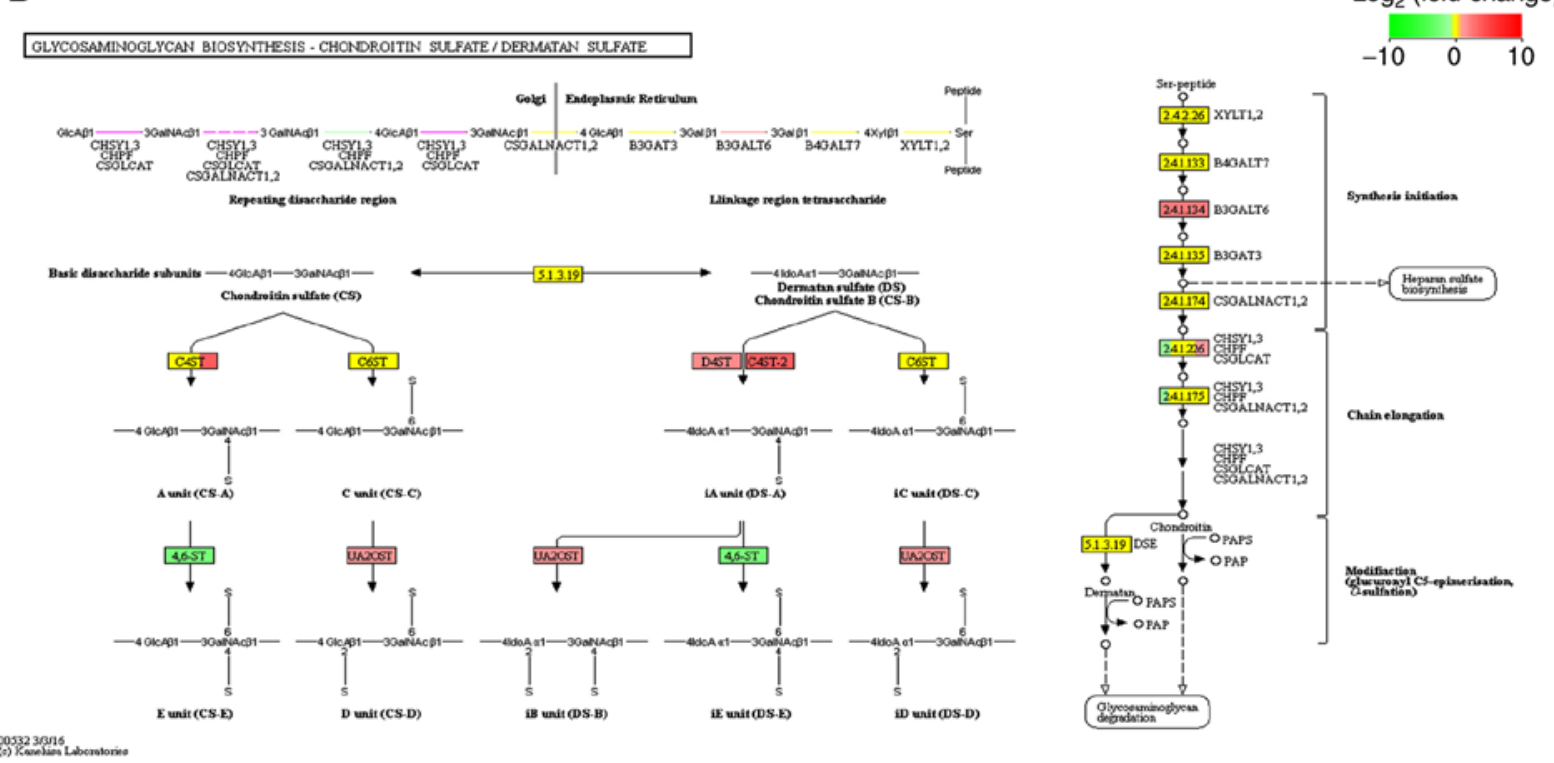

Figure 6. (A) KEGG enrichment analysis. The horizontal axis represents the Rich factor; the higher the value, the higher the enrichment. The vertical axis represents the pathway terms with high enrichment. The q-value is represented by the color; the redder the color, the smaller the q-value, indicating that the enrichment was more notable. The size of the dot indicates the number of differentially expressed genes in this term. Only the top 30 KEGG pathways with the highest degree of enrichment were analyzed. (B) Glycosaminoglycan biosynthesis-chondroitin sulfate/dermatan sulfate pathway. 
C

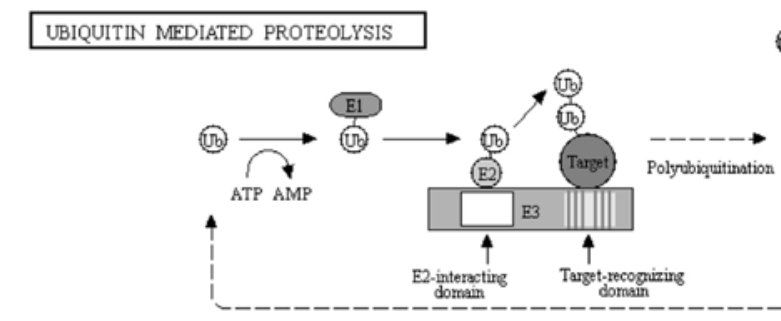

(Ubiquitin-activating enzyme) UBE1 URLFIA UBLE1B UBE1C

HECT type E3

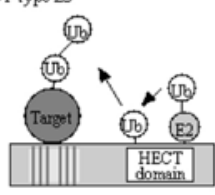

\begin{tabular}{|l|l|l|l|l|l|}
\hline E6AP UBE3B UBE3C Smurf Itch \\
\hline
\end{tabular} \begin{tabular}{|l|l|l|l|l|}
\hline WWP1 & WWP2 & TRIP12 & NEDD4 & ARF-BP1 \\
\hline NW
\end{tabular}

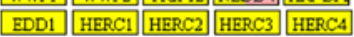

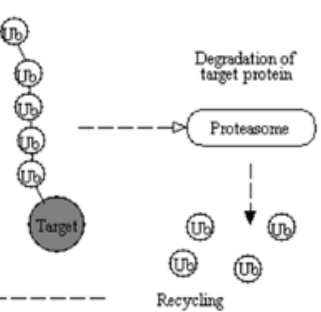

(Ubiquitim-conjugating enzyme)

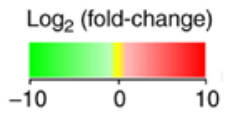

multi subunit RING-finger type E3

Cullin-Rbx E3

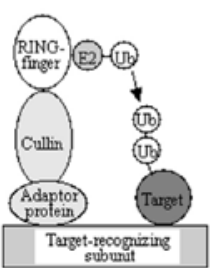

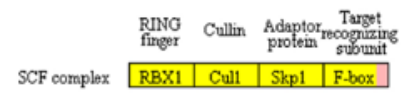

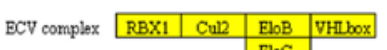

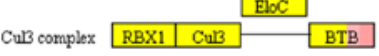

\begin{tabular}{|l|l|l|l|l|}
\hline Cul4 complex & RBX1 & Cul4 & DDB1 & DCAF \\
\cline { 2 - 4 }
\end{tabular}

\begin{tabular}{|l|l|l|l|l|}
\hline ECS complox & RBX2 & Culs & EloB & socsoox \\
\cline { 2 - 5 }
\end{tabular}

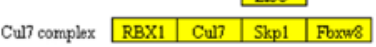

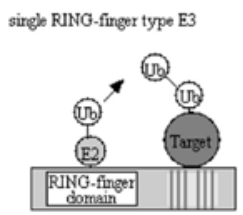

\begin{tabular}{|l|l|l|l|l|l|}
\hline Mdm2 CBL & Parkin SIAH.1 & PML TRAF6 MEKK \\
\hline
\end{tabular} \begin{tabular}{|l|l|l|l|l|l|l|l|l|}
\hline COP1 & PIRH2 & CIAPs & PIAS & SVYN & NHLRC1 & AIRE \\
\hline
\end{tabular} \begin{tabular}{ll|l|l|l|l|l|l|l|l|l|l|l}
\hline MGRNI & BRCA1 & FANCL & MID1 & Trim32 & Trim3? \\
\hline
\end{tabular}

04120 5,20/16
(c) Kanehisa Loboratonies

D

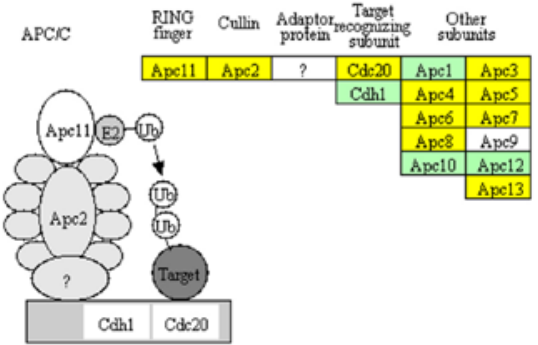
BLADDER CANCER

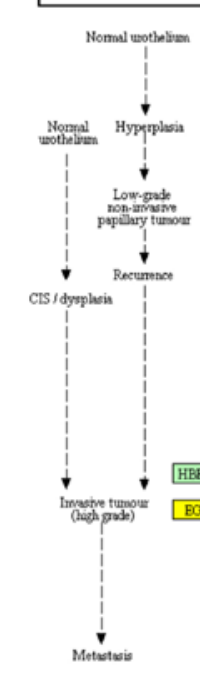

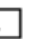
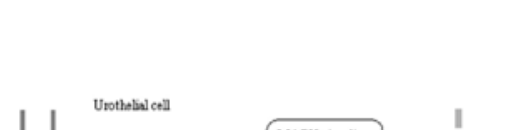
Mono-ADP-ribosylation serves varying roles in a number of biological processes, and its effects are dependent on the location of the modification within the protein. Identification of ADP-ribosylated proteins and ADP-ribose acceptor sites is important for understanding the biological function of ADP ribosylation $(32,33)$. Histones are known to be targets of ADP ribosylation; however, there is a dearth of studies identifying specific sites (34). In a previous study, it was shown that the H3R117 site in Lovo cells is ADP-ribosylated using LC-MS/MS, and there are numerous studies examining the effects of this specific ribosylation. Mono-ADP-ribosylation may affect CRC by modulating enzyme activity or gene expression of associated enzymes (14,35). As CRC is very heterogeneous and has multiple potential evolutionary paths (36), and as mono-ADP-ribosylation of H3R117 is closely associated with CRC, it was hypothesized that changes in the mono-ADP-ribosylation of H3R117 in specific genes in CRC cells may affect downstream pathways associated with the proteins encoded by these genes. Therefore, transcriptome sequencing and bioinformatics were used to investigate the potential biological functions of mono-ADP-ribosylation of H3R117 in gene expression and the associated pathways in CRC.

High quality raw data was obtained by sequencing, and quality controls were used to ensure the accuracy of the data. Subsequently, bioinformatics analysis was used to determine changes in genes and the relevance of these to the development and progression of CRC following mutation of the mono-ADP-ribosylation site. The results showed that a total of 558,174 DEGs were identified, of which 2,324 were considered significant. Based on the results of the differential expression analysis, a Venn diagram was used to visually show the number of co-expressed and uniquely expressed genes in each sample. The protein-protein interaction network highlighted potential interactions associated with these DEGs. Furthermore, the sequencing results showed that the effect of mono-ADP-ribosylation on CRC was very complex, and affected various functions and pathways.

A large number of SNP/indel sites were screened in both the untransfected cells and cells transfected with the mutant construct, and it was shown that the expression patterns of the two samples were similar, with both groups possessing a large number of SNPs. When examining the SNPs, $A>G$ and $\mathrm{T}>\mathrm{C}$ were considered more prominent. For AS, TSS, SKIP and TTS were the three types of AS that occurred more frequently. The presence of SNP sites is associated with tumor susceptibility (37). Studies have identified common SNPs associated with long-term risk and timing of metastasis of CRC in Caucasian patients with stage I-III low microsatellite instability/microsatellite stable cancer (38), and the AS pattern of mRNAs differ between the tumor and the normal tissue from which it originated (39). Abnormal AS is a common phenomenon associated with cancer progression, and is also a characteristic of CRC (40). In the present study, the types and characteristics of variations in genetic structure prior to and following mutation of the mono-ADP-ribosylation site was assessed in CRC cells, laying a foundation for further analysis and research.

GO analysis showed that these DEGs were enriched in pathways associated with molecular function, cellular component and biological process. In the biological process category, pathways associated with 'metabolic process' and 'negative regulation of biological process' were most frequently observed. In the cellular component category, pathways associated with 'organelle' and 'organelle part' and 'membrane-enclosed lumen' were most frequently observed. In molecular function, pathways associated with 'binding' and 'catalytic activity' were the most frequently observed. The mapping of DEGs using KOG annotation showed that only chromatin structure and dynamics $(q<0.05)$ differed significantly. A total of 43 genes were involved in the annotation classification of KOG, among these, upregulated genes were more common than downregulated genes, compared with the untransfected cells. The genes with the greatest fold change were HIST2H3PS2, HIST2H3D, HIST1H2AI and CENPBD1.

Nuclear structure is the most important histological feature that distinguishes cancer cells from normal cells (41). In CRC, the most common type of genomic instability is chromosomal instability, which results in persistent quantitative and structural chromosomal aberrations in cancer cells, leading to intracellular heterogeneity (42), and accounts for $\sim 85 \%$ of all sporadic cases of CRC (43). Activation of oncogenes and inactivation of tumor suppressor genes are caused by various types of somatic gene mutations, including variations in chromatin structure (44). A previous study showed that the H3R117A mutation promoted the transcription and expression of the ten-eleven translocation (TET) family member TET1, as its chromatin was made more accessible by the mutation (35). Studies have shown that lipopolysaccharide stimulation induces histone ADP-ribosylation at transcriptionally active and accessible chromatin regions in macrophages (45). Both mono-ADP-ribosylation and poly-ADP-ribosylation directly affects chromatin structure (46). Therefore mono-ADP-ribosylation of H3R117 may be involved in the development of CRC by affecting the structure and dynamics of chromatin. Based on this, it was shown that the histone variants HIST2H3PS2, HIST2H3D, HIST1H2AI and CENPBD1 were significantly differentially expressed In eukaryotes, the existence of histone variants is based on differences in the primary amino acid sequences, and these variants have different regulatory mechanisms of expression, deposition and genome occupancy (33). Histone variants can alter the structure of nucleosomes and participate in the transcription, replication and repair of DNA (47). CENPBD1 contributes to the formation of the centromere, and may be associated with the prognosis of head and neck squamous cell carcinoma (48). However, their role in CRC has not been determined. Therefore, further experimental studies are required to determine their relevance.

KEGG enrichment analysis screened 306 pathways; among these, the glycosaminoglycan biosynthesis-chondroitin sulfate/dermatan sulfate pathway was the most significantly different. In addition, there were other pathways which were significantly different which were investigated in previous studies, including 'cell cycle progression' and 'apoptosis' $(14,49)$. In the present study, a focus was placed on glycosaminoglycan biosynthesis-chondroitin sulfate/dermatan sulfate. Glycosaminoglycan is an important component of the extracellular matrix. There are four types of glycosaminoglycans: Hyaluronan, heparin/heparan sulfate, chondroitin sulfate/dermatan sulfate and keratin sulfate. Furthermore, chondroitin sulfate is divided into four different classes: Chondroitin sulfate A, C, D and E. Chondroitin sulfate B is 
the old name for dermatan sulfate, but it is no longer classified a chondroitin sulfate (50).

Chondroitin sulfate/dermatan sulfate proteoglycans exert their functions by interacting with specific proteins, such as growth factors, matrix proteins and cell surface receptors, and they serve an important role in cancer (28). Dermatan sulfate epimerase catalyzes the conversion of chondroitin sulfate to dermatan sulfate, and is considered an important mediator of the development of malignant characteristics in hepatocellular carcinoma (51). The metabolism of chondroitin sulfate/dermatan sulfate in tumor stroma is strongly altered. In certain types of cancer, including CRC, the stromal content of dermatan sulfate is increased, and the stromal accumulation of chondroitin-6-sulfate affects the progression and metastasis of the tumor (52). Similarly, chondroitin sulfate serves an important role in the metastasis of breast cancer (53). Increased chondroitin sulfate chain length promotes chemoresistance and epithelial-mesenchymal transition during the progression of CRC (22), and the structure of chondroitin sulfate/dermatan sulfate is significantly altered compared with normal tissue in colon cancer (18). A variety of glycosyltransferases and sulfotransferases are involved in the biosynthesis of chondroitin sulfate, and alterations of the qualitative and quantitative terms of glycosaminoglycans are attributed to changes in enzymes (18). Additionally, it has been reported that chondroitin sulfate/dermatan sulfate proteoglycans present at the cell surface interact with Wnt, resulting in increased accumulation of $\beta$-catenin, thus affecting tumor metastasis (28). In CRC, it has been hypothesized that the Wnt pathway interacts with the chondroitin sulfate/dermatan sulfate components of the CRC stroma (54). Metastasis is the leading cause of mortality in CRC, and aberrant activation of the Wnt signaling serves a crucial role in all stages of CRC development and progression, including metastasis (55). In the present study, 7 genes were enriched in the glycosaminoglycan biosynthesis-chondroitin sulfate/dermatan sulfate pathway. These were CHPF (-1.2367585245), B3GALT6 (2.33805834947), CHPF2 (1.10174702944), CHST12 (3.60850410389), CHST14 (1.60630204961), UST (1.34378885046) and CHST15 (-2.4063182915). Among these, B3GALT6, CHPF2, CHST12, CHST14 and UST were upregulated, and CHPF and CHST15 were downregulated. RT-qPCR was used to verify the top three DEGs based on fold change. The experimental results were consistent with the sequencing results. Furthermore, western blotting and immunofluorescence experiments showed that $\beta$-catenin expression was significantly reduced in the mutant group. Based on these results, it was hypothesized that mono-ADP-ribosylation of histone regulated the structure or expression of chondroitin sulfate by regulating the synthesis of glycosaminoglycan-related enzymes, and subsequently interfering with $\mathrm{Wnt} / \beta$-catenin signaling to affect $\mathrm{CRC}$ metastasis. However, the specific alterations in chondroitin sulfate/dermatan sulfate chains were not determined. Therefore, the role of this pathway in the development of tumors requires further study, and the data show that mono-ADP-ribosylation may affect this pathway via an unknown mechanism.

The second significant pathway was the ubiquitin-mediated proteolysis pathway. One of the proteolytic systems of eukaryotic cells is the ubiquitin-proteasome system (USP). USP is a selective proteolytic system in which substrates are identified and labeled with ubiquitin to mark them for degradation by the protease system (56). The estrogen receptor (ER) is considered the most successful molecular target of cancer drug discovery; however, ER $\alpha$ is not detected in $>60 \%$ of breast and ovarian cancers. A possible reason for its deletion may be the coupling of ER target gene transcription with receptor proteolysis. ER is rapidly ubiquitinated and degraded after estrogen binding (57). Studies have identified 40 support vector machine-classified signature genes in metastatic CRC, and when KEGG enrichment was performed on these genes, one of the most significantly enriched pathways was the ubiquitin-mediated proteolysis pathway (58). The USP also serves an important role in transcriptional regulation (57). The results of the present study showed that the ubiquitin-mediated proteolysis pathway was the second most significantly enriched pathway based on differential gene expression data, suggesting that mono-ADP-ribosylation may serve a role in CRC in this pathway, and thus the detailed mechanisms of the involvement of mono-ADP-ribosylation in ubiquitin-mediated degradation require further study.

The third significant pathway was the bladder cancer pathway. This suggested that mono-ADP-ribosylation may affect the expression of genes involved in a variety of tumors, which will not be discussed in depth here.

Mono-ADP-ribosylation serves an important role in $\mathrm{CRC}$, the exact mechanisms of which are being discovered. In the present study, transcriptome sequencing technology was used to analyze the DEGs following the alteration of mono-ADP-ribosylation modifications at specific sites. A series of structural variations were determined to be associated with functional differences and pathway alterations, providing a theoretical basis for studying the effect of mono-ADP-ribosylation in CRC. Identifying potential targets for the treatment of CRC from the perspective of epigenetics may result in novel therapeutic options for the treatment of patients.

\section{Acknowledgements}

We are very grateful to Dr Yi Tang and Dr Ming Li of the Department of Pathology, Molecular Medicine and Cancer Research Center (Chongqing Medical University, China) for their work in writing and modifying the manuscript.

\section{Funding}

The research was supported by Innovation Project of Graduate Student in Chongqing (grant no. CYB17100); Scientific Research Foundation of Chongqing Medical University (grant no. 201413); National Nature Science Foundation of China (grant no. 30870946); Science and Technology Plan Project of Yuzhong District in Chongqing (grant no. 20140106); and The National High Technology Research and Development Program of China (grant no. 2012AA02A201).

\section{Availability of data and materials}

The datasets used and/or analyzed during the present study are available from the corresponding author on reasonable request.

\section{Authors' contributions}

NNZ and TL performed the cell culture and RNA extraction. YLW designed and conducted the experiments. MX, QSL, 
LY, XL and CLW participated in analyzing the sequencing results.

\section{Ethics approval and consent to participate}

Not applicable.

\section{Patient consent for publication}

Not applicable.

\section{Competing interests}

The authors declare that they have no competing interests.

\section{References}

1. Araghi M, Soerjomataram I, Jenkins M, Brierley J, Morris E, Bray $\mathrm{F}$ and Arnold M: Global trends in colorectal cancer mortality: Projections to the year 2035. Int J Cancer 144: 2992-3000, 2019.

2. Schreuders EH, Ruco A, Rabeneck L, Schoen RE, Sung JJ, Young GP and Kuipers EJ: Colorectal cancer screening: A global overview of existing programmes. Gut 64: 1637-1649, 2015

3. Li XX, Peng JJ, Liang L, Huang LY, Li DW, Shi DB, Zheng HT and Cai SJ: RNA-seq identifies determinants of oxaliplatin sensitivity in colorectal cancer cell lines. Int J Clin Exp Pathol 7: 3763-3770, 2014.

4. Cohen MS and Chang P: Insights into the biogenesis, function, and regulation of ADP-ribosylation. Nat Chem Biol 14: 236-243, 2018.

5. Luscher B, Butepage M, Eckei L, Krieg S, Verheugd P and Shilton BH: ADP-ribosylation, a multifaceted posttranslational modification involved in the control of cell physiology in health and disease. Chem Rev 118: 1092-1136, 2018.

6. Jain PG and Patel BD: Medicinal chemistry approaches of poly ADP-Ribose polymerase 1 (PARP1) inhibitors as anticancer agents-A recent update. Eur J Med Chem 165: 198-215, 2019.

7. Kunze FA and Hottiger MO: Regulating immunity via ADP-ribosylation: Therapeutic implications and beyond. Trends Immunol 40: 159-173, 2019.

8. Hendriks IA, Larsen SC and Nielsen ML: An advanced strategy for comprehensive profiling of ADP-ribosylation sites using mass spectrometry-based proteomics. Mol Cell Proteomics 18: 1010-1026, 2019

9. Sakthianandeswaren A, Parsons MJ, Mouradov D, MacKinnon RN, Catimel B, Liu S, Palmieri M, Love C, Jorissen RN, Li S, et al: MACROD2 haploinsufficiency impairs catalytic activity of PARP1 and promotes chromosome instability and growth of intestinal tumors. Cancer Discov 8: 988-1005, 2018

10. Rajaram M, Zhang J, Wang T, Li J, Kuscu C, Qi H, Kato M, Grubor V, Weil RJ, Helland A, et al: Two distinct categories of focal deletions in cancer genomes. PLoS One 8: e66264, 2013.

11. Zhang Y, Wang J, Ding M and Yu Y: Site-specific characterization of the Asp- and Glu-ADP-ribosylated proteome. Nat Methods 10: 981-984, 2013.

12. Bonfiglio JJ, Fontana P, Zhang Q, Colby T, Gibbs-Seymour I, Atanassov I, Bartlett E, Zaja R, Ahel I and Matic I: Serine ADP-ribosylation depends on HPF1. Mol Cell 65: 932-940 e6, 2017.

13. Paone G, Wada A, Stevens LA, Matin A, Hirayama T, Levine RL and Moss J: ADP ribosylation of human neutrophil peptide-1 regulates its biological properties. Proc Natl Acad Sci USA 99: 8231-8235, 2002.

14. Ling F, Tang Y, Li M, Li QS, Li X, Yang L, Zhao W, Jin CC, Zeng Z, Liu C, et al: Mono-ADP-ribosylation of histone 3 at arginine-117 promotes proliferation through its interaction with P300. Oncotarget 8: 72773-72787, 2017.

15. Jing C, Ma R, Cao H, Wang Z, Liu S, Chen D, Wu Y, Zhang J and $\mathrm{Wu} \mathrm{J}$ : Long noncoding RNA and mRNA profiling in cetuximab-resistant colorectal cancer cells by RNA sequencing analysis. Cancer Med 8: 1641-1651, 2019.

16. Vacchelli E, Ma Y, Baracco EE, Sistigu A, Enot DP, Pietrocola F, Yang H, Adjemian S, Chaba K, Semeraro M, et al: Chemotherapy-induced antitumor immunity requires formyl peptide receptor 1. Science 350: 972-978, 2015.
17. Lin KT, Shann YJ, Chau GY, Hsu CN and Huang CY: Identification of latent biomarkers in hepatocellular carcinoma by ultra-deep whole-transcriptome sequencing. Oncogene 33 : 4786-4794, 2014

18. Kalathas D, Theocharis DA, Bounias D, Kyriakopoulou D, Papageorgakopoulou N, Stavropoulos MS and Vynios DH: Chondroitin synthases I, II, III and chondroitin sulfate glucuronyltransferase expression in colorectal cancer. Mol Med Rep 4: 363-368, 2011.

19. Kalathas D, Theocharis DA, Bounias D, Kyriakopoulou D, Papageorgakopoulou N, Stavropoulos MS and Vynios DH: Alterations of glycosaminoglycan disaccharide content and composition in colorectal cancer: Structural and expressional studies. Oncol Rep 22: 369-375, 2009.

20. Jayson GC, Lyon M, Paraskeva C, Turnbull JE, Deakin JA and Gallagher JT: Heparan sulfate undergoes specific structural changes during the progression from human colon adenoma to carcinoma in vitro. J Biol Chem 273: 51-57, 1998.

21. Zeng L, Qian J, Luo X, Zhou A, Zhang Z and Fang Q: CHSY1 promoted proliferation and suppressed apoptosis in colorectal cancer through regulation of the $\mathrm{NF \kappa B}$ and/or caspase- $3 / 7$ signaling pathway. Oncol Lett 16: 6140-6146, 2018.

22. Hoshiba T: An extracellular matrix (ECM) model at high malignant colorectal tumor increases chondroitin sulfate chains to promote epithelial-mesenchymal transition and chemoresistance acquisition. Exp Cell Res 370: 571-578, 2018.

23. Bolger AM, Lohse $M$ and Usadel B: Trimmomatic: A flexible trimmer for Illumina sequence data. Bioinformatics 30: 2114-2120, 2014

24. Pertea M, Pertea GM, Antonescu CM, Chang TC, Mendell JT and Salzberg SL: StringTie enables improved reconstruction of a transcriptome from RNA-seq reads. Nat Biotechnol 33: 290-295, 2015.

25. Livak KJ and Schmittgen TD: Analysis of relative gene expression data using real-time quantitative PCR and the 2(-Delta Delta $\mathrm{C}(\mathrm{T}))$ method. Methods 25: 402-408, 2001.

26. Okonechnikov K, Conesa A and García-Alcalde F: Qualimap 2: Advanced multi-sample quality control for high-throughput sequencing data. Bioinformatics 32: 292-294, 2016.

27. Kanehisa M, Furumichi M, Tanabe M, Sato Y and Morishima K: KEGG: New perspectives on genomes, pathways, diseases and drugs. Nucleic Acids Res 45 (D1): D353-D361, 2017.

28. Mizumoto S, Yamada S and Sugahara K: Molecular interactions between chondroitin-dermatan sulfate and growth factors/receptors/matrix proteins. Curr Opin Struct Biol 34: 35-42, 2015.

29. Yoon YS, Kim JW, Kang KW, Kim YS, Choi KH and Joe CO: Poly(ADP-ribosyl)ation of histone $\mathrm{H} 1$ correlates with internucleosomal DNA fragmentation during apoptosis. J Biol Chem 271: 9129-9134, 1996.

30. Voigt P, Tee WW and Reinberg D: A double take on bivalent promoters. Genes Dev 27: 1318-1338, 2013.

31. Gupte R, Liu Z and Kraus WL: PARPs and ADP-ribosylation: Recent advances linking molecular functions to biological outcomes. Genes Dev 31: 101-126, 2017.

32. Hottiger MO: Nuclear ADP-ribosylation and its role in chromatin plasticity, cell differentiation, and epigenetics. Annu Rev Biochem 84: 227-263, 2015.

33. Xu YM, Du JY and Lau AT: Posttranslational modifications of human histone H3: An update. Proteomics 14: 2047-2060, 2014.

34. Rakhimova A, Ura S, Hsu DW, Wang HY, Pears CJ and Lakin ND: Site-specific ADP-ribosylation of histone H2B in response to DNA double strand breaks. Sci Rep 7: 43750, 2017.

35. Li M, Tang Y, Li Q, Xiao M, Yang Y and Wang Y: Mono-ADPribosylation of H3R117 traps 5mC hydroxylase TET1 to impair demethylation of tumor suppressor gene TFPI2. Oncogene 38: 3488-3503, 2019. Castle JC, Loewer M, Boegel S, de Graaf J, Bender C, Tadmor AD, Boisguerin V, Bukur T, Sorn P, Paret C, et al: Immunomic, genomic and transcriptomic characterization of CT26 colorectal carcinoma. BMC Genomics 15: 190, 2014.

36. Simonian M, Mosallaei M, Khosravi S and Salehi R: rs12904 polymorphism in the 3'-untranslated region of ephrin A1 ligand and the risk of sporadic colorectal cancer in the Iranian population. J Cancer Res Ther 15: 15-19, 2019.

37. Penney ME, Parfrey PS, Savas S and Yilmaz YE: A genome-wide association study identifies single nucleotide polymorphisms associated with time-to-metastasis in colorectal cancer. BMC Cancer 19: 133, 2019.

38. Sveen A, Kilpinen S, Ruusulehto A, Lothe RA and Skotheim RI: Aberrant RNA splicing in cancer; expression changes and driver mutations of splicing factor genes. Oncogene 35: 2413-2427, 2016. 
39. Liu J, Li H, Shen S, Sun L, Yuan Y and Xing C: Alternative splicing events implicated in carcinogenesis and prognosis of colorectal cancer. J Cancer 9: 1754-1764, 2018.

40. Resar L, Chia L and Xian L: Lessons from the Crypt: HMGA1-Amping up Wnt for stem cells and tumor progression. Cancer Res 78: 1890-1897, 2018.

41. Lee AJ, Endesfelder D, Rowan AJ, Walther A, Birkbak NJ, Futreal PA, Downward J, Szallasi Z, Tomlinson IP, Howell M, et al: Chromosomal instability confers intrinsic multidrug resistance. Cancer Res 71: 1858-1870, 2011.

42. Ahmed D, Eide PW, Eilertsen IA, Danielsen SA, Eknæs M, Hektoen M, Lind GE and Lothe RA: Epigenetic and genetic features of 24 colon cancer cell lines. Oncogenesis 2: e71, 2013.

43. van den Broek E, Dijkstra MJ, Krijgsman O, Sie D, Haan JC, Traets JJ, van de Wiel MA, Nagtegaal ID, Punt CJ, Carvalho B, et al: High prevalence and clinical relevance of genes affected by chromosomal breaks in colorectal cancer. PLoS One 10: e0138141, 2015.

44. Martinez-Zamudio R and Ha HC: Histone ADP-ribosylation facilitates gene transcription by directly remodeling nucleosomes. Mol Cell Biol 32: 2490-2502, 2012.

45. Abplanalp J and Hottiger MO: Cell fate regulation by chromatin ADP-ribosylation. Semin Cell Dev Biol 63: 114-122, 2017.

46. Clouaire T and Legube G: A snapshot on the Cis chromatin response to DNA double-strand breaks. Trends Genet 35: 330-345, 2019.

47. Wintergerst L, Selmansberger M, Maihoefer C, Schüttrumpf L, Walch A, Wilke C, Pitea A, Woischke C, Baumeister P, Kirchner T, et al: A prognostic mRNA expression signature of four 16q24.3 genes in radio(chemo)therapy-treated head and neck squamous cell carcinoma (HNSCC). Mol Oncol 12: 2085-2101, 2018.

48. Zhen Z: Effects of Histone H3 Arginine-specific Mono-ADPribosylation on proliferation and apoptosis of human colon cancer cell and its possible mechanism. Chongqing Med Univ, 2016.
49. Mende M, Bednarek C, Wawryszyn M, Sauter P, Biskup MB, Schepers U and Bräse S: Chemical synthesis of glycosaminoglycans. Chem Rev 116: 8193-8255, 2016.

50. Liao WC, Yen HR, Liao CK, Tseng TJ, Lan CT and Liu CH: DSE regulates the malignant characters of hepatocellular carcinoma cells by modulating CCL5/CCR1 axis. Am J Cancer Res 9: 347-362, 2019.

51. Pudelko A, Wisowski G, Olczyk K and Kozma EM: The dual role of the glycosaminoglycan chondroitin-6-sulfate in the development, progression and metastasis of cancer. FEBS J 286: 1815-1837, 2019.

52. Kopec M, Imiela A and Abramczyk H: Monitoring glycosylation metabolism in brain and breast cancer by Raman imaging. Sci Rep 9: 166, 2019.

53. Nikitovic D, Chatzinikolaou G, Tsiaoussis J, Tsatsakis A, Karamanos NK and Tzanakakis GN: Insights into targeting colon cancer cell fate at the level of proteoglycans/glycosaminoglycans. Curr Med Chem 19: 4247-4258, 2012.

54. Qi J, Yu Y, Akilli Öztürk Ö, Holland JD, Besser D, Fritzmann J, Wulf-Goldenberg A, Eckert K, Fichtner I and Birchmeier W: New Wnt/ $\beta$-catenin target genes promote experimental metastasis and migration of colorectal cancer cells through different signals. Gut 65: 1690-1701, 2016.

55. Ji CH and Kwon YT: Crosstalk and interplay between the ubiquitin-proteasome system and autophagy. Mol Cells 40: 441-449, 2017.

56. Zhou W and Slingerland JM: Links between oestrogen receptor activation and proteolysis: Relevance to hormone-regulated cancer therapy. Nat Rev Cancer 14: 26-38, 2014.

57. Zhi J, Sun J, Wang Z and Ding W: Support vector machine classifier for prediction of the metastasis of colorectal cancer. Int J Mol Med 41: 1419-1426, 2018. 